\title{
Pulsation Variables in the AF stars of the Case Low-Dispersion Survey
}

\author{
T.D.Kinman \\ Kitt Peak National Observatory, P.O.Box 26732, Tucson, AZ 85732, U.S.A.
}

\begin{abstract}
The AF stars are those of spectral types $A$ and $F$ that have been discovered on objective-prism plates taken with the Burrell Schmidt in the Case LowDispersion Northern Sky Survey (Pesch and Sanduleak, 1983). In SA 57, this survey is complete to $\mathrm{V}=16.5$. In this field and also in the Lick Astrograph RR Lyrae survey field RR 7 (in the anticenter) the AF stars comprise (a) blue horizontal branch stars and RR Lyrae stars of the halo and (b) stars which have the higher surface gravities of main sequence stars. The two groups can be separated primarily by their differing Balmer jumps and Balmer line-widths. The latter group (which may well include blue stragglers of both Pop I and Pop II) extends some $10 \mathrm{kpc}$ above the galactic plane and shows a wide range of metallicity. Photoelectric photometry of this AF star sample has allowed the detection field RR Lyrae stars of lower-amplitudes than could have been found by conventional blinking techniques; this has led to a significant increase in the number of RR Lyrae stars that are known in SA 57 and RR 7. The cooler main sequence and/or blue straggler AF stars lie in the zone of pulsational instability and one higher-amplitude $\delta$-Scuti star was detected in field RR 7 . It is suggested that these AF stars provide a good sample for studying the incidence of pulsation in the population of older stars that extends beyond the thin disk.
\end{abstract}

\section{Introduction}

The Case Low-Dispersion Northern Sky Survey (Pesch and Sanduleak, 1983 and Pesch, 1991) is being made with the $0.6-\mathrm{m} / 0.9-\mathrm{m} \mathrm{F} / 3.5$ Burrell Schmidt telescope using a $1.8^{\circ} \mathrm{UV}$ transmitting prism $\left(\sim 1000 \AA \mathrm{mm}^{-1}\right.$ at $\mathrm{H}$ and $\left.\mathrm{K}\right)$. The AF stars (stars with spectral types $\mathrm{A}$ and $\mathrm{F}$ ) are those which are described as being in Category IV, IV-V or V in the Case survey. It was originally thought that Category IV and $\mathrm{V}$ corresponded respectively to main sequence and to horizontal branch stars. Later work (Sanduleak, 1989; Kinman, 1992 and MacConnell, Stephenson and Pesch, 1992) has shown, however, that this distinction cannot be made unambiguously from the relatively low $\mathrm{S} / \mathrm{N}$ objective-prism spectra of the fainter stars. Ultraviolet-excess objects of stellar appearance (such as white dwarfs and quasars) can, however, be distinguished in these spectra and so excluded from Categories IV and V. Lists of both Categories of these AF stars are available for regions near the North Galactic 
Pole (NGP) (Sanduleak, 1988 and MacConnell et al., 1992). Category V stars alone have been published for a large region between the Anticenter and the NGP (Pesch and Sanduleak, 1989). I was first interested in the AF stars because they included nearly all the RR Lyrae stars (brighter than $B \sim 17$ ) that had been discovered by blinking plates taken with the Lick Astrograph at the NGP (Kinman, Wirtanen and Janes, 1966). Sanduleak kindly made available to me the identifications of the stars of both categories in SA 57 at the NGP (which is also Lick Astrograph field RR 4) and also in the Lick Astrograph field RR $7\left(1=183^{\circ} ; b=+37^{\circ}\right)$ in the Anticenter. The field of SA 57 is important because it has been included in a number of other surveys - in particular the multicolor survey of Stobie and Ishida (1987). A comparison with this survey showed that the Case survey in SA 57 is complete to $\mathrm{V}=16.5$ within the range $0.0 \leq(B-V) \leq 0.32$ (Kinman, 1992). More recent work suggests that the red limit of the Case survey may vary from region to region and this is also probably true of the bright limit.

The importance of the Case AF stars is that they provide a moderately deep survey for probing both the halo (with the RR Lyrae stars, blue horizontal branch stars and possibly the halo blue stragglers) and also the stars of the extended or "thick" disk. In the case of the halo, the use of the AF stars allows one to determine the horizontal-branch morphology from the relative numbers of bhb to RR Lyrae stars and so puts an interesting constraint on the nature of this population.

\section{Observational techniques for identifying blue horizontal branch (bhb)} stars

The characteristic variability of the RR Lyrae stars - the bhb stars that lie in the zone of pulsational instability $\left(0.20 \leq(\mathrm{B}-\mathrm{V})_{0} \leq 0.40\right)$ - makes them relatively easy to identify. It is those with $(B-V)_{0} \leq 0.20$ and particularly those with $(B-V)_{0} \sim 0.0$ that are more difficult to classify with certainty. The two fields (SA 57 and RR 7) contain some $150 \mathrm{AF}$ stars and the majority of these are fainter than $\mathrm{V}=14$. The well-known technique of separating bhb stars from higher-gravity main-sequence stars using the $c_{1}$ index of Stromgren photometry was not practical for such faint stars with the equipment available. A hybrid $(\mathrm{u}-\mathrm{B}$ ) index (where $\mathrm{u}$ is a Stromgren filter) can, however, be used to measure the Balmer jump and the separation can be made in a $(\mathrm{u}-\mathrm{B})$ vs. (B-V) plot (Kinman, 1992). Bidelman (1992) has noted that, in addition to the bhb stars, somewhat evolved normal early-type stars can also have a high $c_{1}$ index. Corbally and Gray (1992) have used high S/N CCD spectra to classify 67 of the field horizontal branch stars listed by Philip (1984) and conclude that only two are actually field horizontal branch stars. This conclusion may need modification. Their spectral classification of the classic prototype field horizontal branch star HD 161817 (Oke, Greenstein and Gunn, 1966) which has a radial velocity of $-363 \mathrm{~km} / \mathrm{s}$ and $[\mathrm{Fe} / \mathrm{H}]=-1.7$ (Adelman and Hill, 1987), as an A8 dwarf with weak metal lines does not agree with our assessment. HD 161817 has a $(B-V)_{0}$ of $\sim 0.13$ which is close to that of an $\mathrm{A} 3 \mathrm{~V}$ star, but its Balmer lines are much narrower and its ultraviolet continuum quite different from such a star (Fig. 1). The subject needs further 
for all stars.

A provisional analysis showed 15 stars in which there seemed to be a significant difference between the metallicity derived from the $\mathrm{K}$-line and that derived from the $\mathrm{Mg}$ II $\lambda 4481$ line. In 13 of these cases, the metallicity derived from the Fe I $\lambda 4271$ line was in better agreement with that found from the $\mathrm{Mg}$ II line than that derived from the K-line.

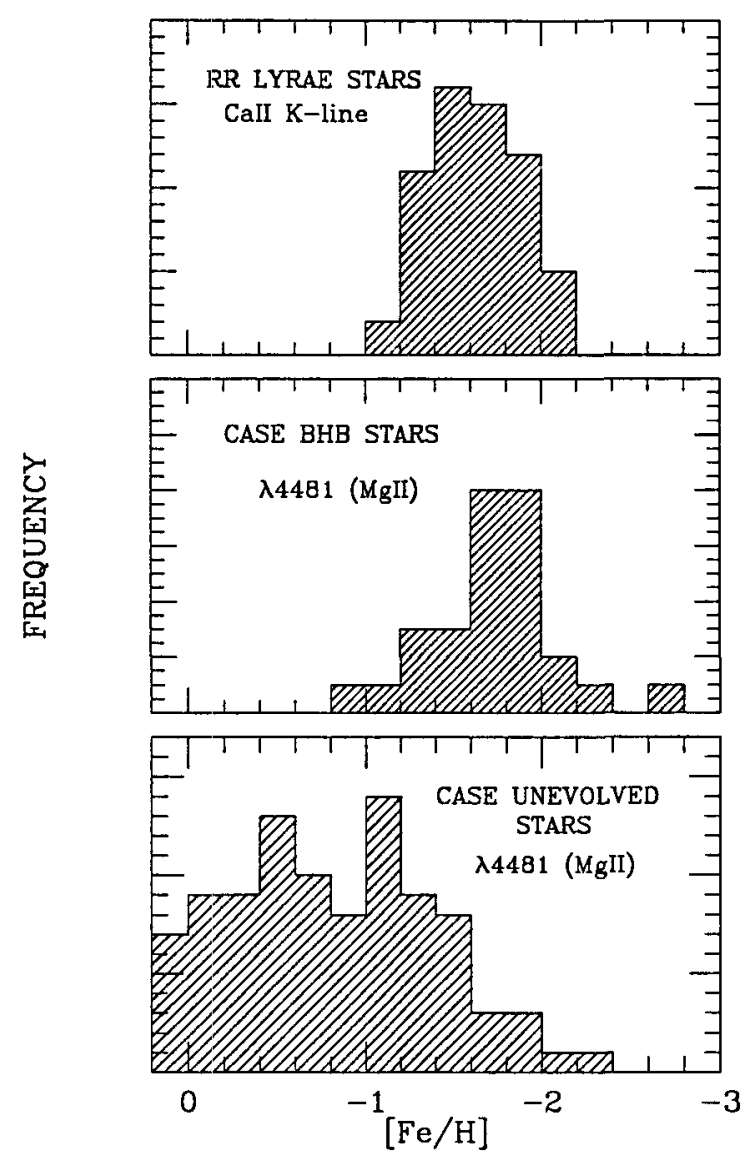

Figure 2 The upper panel shows the distribution of $[\mathrm{Fe} / \mathrm{H}]$ derived from the $\mathrm{Ca}$ II K-line ( $\Delta \mathrm{S}$ method) for the RR Lyrae stars outside the solar circle (Suntzeff, Kinman and Kraft, 1990). The middle panel shows the distribution of $[\mathrm{Fe} / \mathrm{H}]$ for the Case AF stars that are judged to be bhb stars on the basis of their $(\mathrm{u}-\mathrm{B})$ index; and the lower panel gives the distribution for those that are not. In both lower and middle panels, $[\mathrm{Fe} / \mathrm{H}]$ is derived from provisional measurements of the $\mathrm{Mg}$ II $\lambda 4481$ line.

The distribution of the values of $[\mathrm{Fe} / \mathrm{H}]$ for the AF stars in the two fields (SA 57 and RR 7) derived from these provisional measurements of the $\mathrm{Mg}$ II $\lambda 4481$ line are 
shown in Fig 2 . The middle panel shows the distribution for the bhb stars; the lower panel shows the distribution for the remaining (unevolved) stars. Two stars for which the $\mathrm{Mg}$ II line gives $[\mathrm{Fe} / \mathrm{H}]$ of +0.5 and +0.7 have been omitted from the histogram in the lowest panel; one of these stars is a known Am star. The top panel in Fig. 2 shows the distribution of $[\mathrm{Fe} / \mathrm{H}]$ for the RR Lyrae variables outside the solar circle (Suntzeff, Kinman and Kraft, 1990). The RR Lyrae abundances were derived from the Ca II K-line using Preston's $\Delta S$ method. Bearing in mind the measuring errors that must be present in the equivalent widths of the $\mathrm{Mg}$ II line, the agreement between the $[\mathrm{Fe} / \mathrm{H}]$ distributions of the two halo samples is as satisfactory as could be expected. Very provisional radial velocities were determined for the stars in SA 57. In this field the radial velocity dispersion is nearly the same as the z-motion dispersion and is $\pm 136 \mathrm{~km} / \mathrm{s}$ for the bhb stars. For the remaining or "unevolved" AF stars, those with $[\mathrm{Fe} / \mathrm{H}] \geq-1.0$ have a dispersion of $\pm 48 \mathrm{~km} / \mathrm{s}$ and those with $[\mathrm{Fe} / \mathrm{H}] \leq-1.0$ a dispersion of $\pm 70 \mathrm{~km} / \mathrm{s}$. These velocity dispersions are probably inflated by significant errors but valid enough to show that the stars with weak $\mathrm{Mg}$ II $\lambda 4481$ which have been assigned $[\mathrm{Fe} / \mathrm{H}] \leq-1.0$ are not low-velocity Population I $\lambda$ Boo stars.

\section{Pulsation variables in the AF stars}

Some of the AF stars populate the instability strip. Photoelectric monitoring of a sample of AF stars affords a practical way of picking out the pulsation variables even
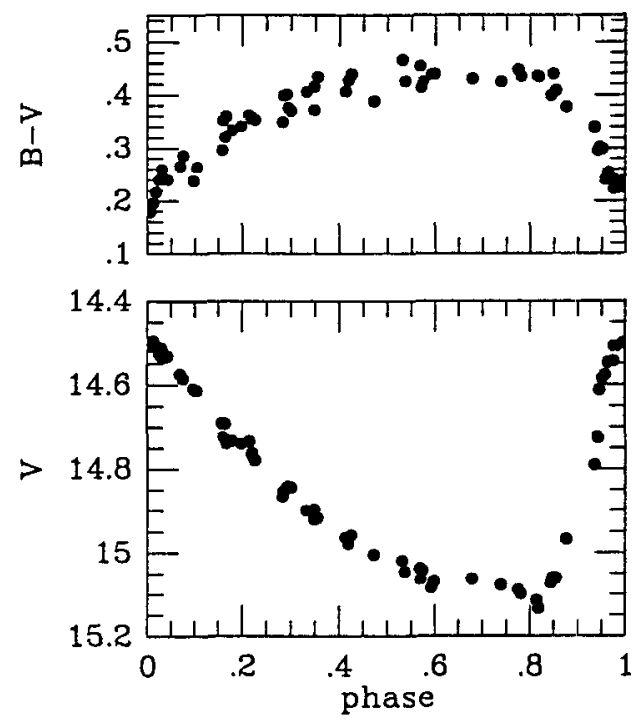

Figure 3 The light curves of the Case star AF 167 (Sanduleak, 1988). This type ab RR Lyrae star has a period of 0.640648 days.

if they have quite small amplitudes. After discovery, the periods and light curves of the variables can be obtained by further photoelectric observations or by measuring 
the stars on existing survey plates such as those taken with the Lick Astrograph. This is a much more effective way of finding RR Lyrae variables - particularly those of lower amplitude - than by blinking. An example of such a variable is the Case star AF 167 (Sanduleak, 1988). This star lies in the field RR 3 and close to the edge of the overlapping field RR 4 and was missed from their survey by Kinman, Wirtanen and Janes (1966). It is interesting that it is listed (NSV 6031) in the catalogue of stars of suspected variability; it was identified as a possible RR Lyrae star by Kurochkin (1960). It has a blue amplitude (see Fig. 3) of 0.75 magnitudes. This is the amplitude limit at which the surveys with the Lick Astrograph are thought to be complete. Variables with lower amplitude were found - but only with partial completeness. Surveys such as those made with the Lick Astrograph will therefore be incomplete for the lower-amplitude c-type variables. The ratio of the c-type to ab-type variables is one of the parameters that characterizes the Oosterhoff type, and like the ratio of the numbers of RR Lyrae stars to bhb stars is essentially a measure of the way the stars are distributed along the horizontal branch. Twelve new RR Lyrae variables have been discovered so far in SA 57 and RR 7 and work is in progress to determine their periods and light curves.

Amongst the "unevolved stars", a $\delta$ Scuti variable with a B-amplitude of 0.25 magnitudes (period 0.093 days) was found in field RR 7 (GSC 298501044 in the Space Telescope Guide Star Catalog). The star is relatively bright (B 12.2) and its images on the survey plates taken with the Lick Astrograph are very strong; it is therefore not surprising that its variability went undetected. It was possible, however, to make iris astrophotometer measurements of GSC 298501044 on these plates with enough accuracy to produce a light curve. The Astrograph images are of sufficient quality to derive light curves even though the amplitudes are too small for the variables to have been discovered by blinking the plates.

\section{The nature of the non-variable AF stars}

The bhb stars can be convincingly separated out from the other AF stars by observational parameters (Balmer jump, Balmer line widths, etc.) that are related to their lower surface gravity compared with main sequence stars. Undoubtedly there are stars in the disk with high $c_{1}$ index that may mimic these bhb stars. At the height above the plane of most of our bhb star candidates, the ratio of main sequence to halo stars is much smaller than in the solar neighbourhood and so this kind of contamination in our sample is likely to be minimal. We find a metallicity distribution for our bhb sample that is much like that found for RR Lyrae stars in the outer halo and we also (provisionally) find an appropriate velocity dispersion.

The nature of the remaining non-variable stars is less clear and it could well be that they comprise several populations. The merging of other small galaxies with our own is one possiblity (Freeman, 1990). We should also consider mechanisms such as those involving collisions within high-velocity HI clouds (Dyson and Hartquist, 1983) and the ejection of stars from open clusters (Leonard and Duncan, 1990) which are invoked to explain runaway B stars and may also explain some of the extended disk of 
A stars. It has also been suggested that field blue stragglers are merged stars that have been ejected from clusters (Leonard, 1989). Over twenty years ago, Eggen (1969) and later Bond and MacConnell (1971) suggested that the blue stragglers of the old open clusters and the globulars clusters have counterparts in the field; Shields and Twarog (1988) estimated that these field blue stragglers should exist in substantial numbers. That many of these non-bhb AF stars are blue stragglers associated the older stellar populations agrees qualitatively with their having an extended distribution in $\mathrm{z}$ and a wide distribution in metallicity. According to Buonanno, Buzzoni, Corsi, Fusi Pecci and Sandage (1988) the number of blue stragglers and horizontal branch stars in the globular cluster M 3 are rather similar. If this ratio also applies to our sample in SA 57 we should expect to find 3 or 4 halo blue stragglers with $15.0 \leq \mathrm{V} \leq 16.5$. There are 7 non-bhb stars with $[\mathrm{Fe} / \mathrm{H}] \leq-1.4$ in our sample in this magnitude range. Nemec (1989) has reported that $25 \%$ of globular cluster blue stragglers are variable and that most of these are SX Phe stars. It would therefore be interesting to search for this type of variability among the metal-poor non-bhb AF stars.

\section{References:}

Adelman, S.J. and Hill, G., 1987, M.N.R.A.S., 226, 581.

Bidelman, W.P., 1992, in: Luminous High-Latitude Stars, Astr. Soc. of Pacific Conf. Series, to be printed, ed. D. D. Sasselov, Chelsea MI, Astr. Soc. of the Pacific.

Bond, H.E. and MacConnell, D.J., 1971, Astrophys. J., 165, 51.

Buonanno, R., Buzzoni, A., Corsi, C.E., Fusi Pecci F. and Sandage, A.R. 1988, in: I.A.U. Symposium 126, ed. J.E. Grindley and A.G.D. Philip, Dordrecht, Kluwer, p 621.

Corbally, C.J. and Gray, R.O., 1992, in: Peculiar versus Normal Phenomena in A-type and Related stars, ed. F. Castelli and M.M. Dworetsky.

Dyson, J.E. and Hartquist, T.W., 1983, M.N.R.A.S.,203, 1223.

Eggen, O.J. 1969, Publ. Astr. Soc. Pacific, 81, 741.

Freeman, K.C., 1990, in: Dynamics and Interactions of Galaxies, ed. R. Wielen, Springer, Berlin, p. 36.

Gray, R.O., and Garrison, R.F., 1987, Astrophys. J., 65, 581.

Gray, R.O., and Garrison, R.F., 1989, Astrophys. J., 69, 301.

Kinman, T.D., 1992, in: Variable Stars and Galaxies, Astr. Soc. of Pacific Conf. Series,

30, ed. Brian Warner, Chelsea MI, Astr. Soc. of Pacific, p. 19.

Kinman, T.D., Wirtanen, C.A., and Janes, K. 1966, Astrophys. J. Suppl., 13, 379.

Kinman, T.D., Mahaffey, C. and Wirtanen, C.A. 1982, Astron. J., 87, 314.

Kurochkin, H.E., 1960, Variable Stars, 12, 409.

Leonard, P.J.T., 1989, Astron. J., 98, 217.

Leonard, P.J.T. and Duncan, M.J., 1990, Astron. J., 99, 608.

MacConnell, D.J., Stephenson, C.B. and Pesch, P., 1992, Astron. J., (submitted).

Morgan, W.W., 1933, Astrophys. J., 77, 291.

Morrison, H., Flynn, C. and Freeman, K., 1990, Astron. J., 100, 1191.

Nemec, J.M., 1989, in:The use of pulsating variables in fundamental problems in astronomy, ed. E.G. Schmidt, Cambridge Univ. Press, Cambridge, p. 215.

Oke, J.B., Greenstein, J.L. and Gunn, J., 1966, in: Stellar Evolution, ed. R.F. Stein and A.G.W. Cameron, Plenum Press, New York, p. 399. 
Pesch, P., 1991, in: Objective-Prism and Other Surveys: A Meeting in Memory of Nicholas Sanduleak, ed. A.G.D. Philip and A.R. Upgren, L. Davis Philip Press, Schenectady, p. 3.

Pesch, P. and Sanduleak, N., 1983, Astrophys. J. Suppl., 51, 171.

Pesch, P. and Sanduleak, N., 1989, Astrophys. J. Suppl., 71, 549.

Peterson, R.C., Tarbell, T.D. and Carney, B.W., 1983, Astrophys. J., 265, 972.

Philip, A.G.D., 1984, Van Vleck Obs. Contrib., No 2, p. 1.

Pier, J.R., 1983, Astrophys. J. Suppl., 53, 791.

Sanduleak, N., 1988, Astrophys. J. Suppl., 66, 309.

Sanduleak, N., 1989, Astrophys. J. Suppl., 71, 713.

Shields, J.C. and Twarog, B.A., 1988, Astrophy. J., 324, 859.

Slettebak, A., 1954, Astrophy. J., 119, 146.

Stobie, R. and Ishida, K., 1987, Astron. J., 93, 624.

Struve, O. and Wurm, K., 1938, Astrophys. J., 88, 84.

Suntzeff, N.B., Kinman, T.D. and Kraft, R.P., 1991, Astrophys. J., 367, 528.

\section{Discussion:}

M. Breger: You mentioned that the amplitudes of the RR Lyrae stars detected in your survey fields are very low. Are the amplitudes unusually low relative to other field or cluster variables?

T.D.Kinman: Field RR Lyrae stars are usually discovered by blinking survey plates taken with wide-angle Astrographs or Schmidt telescopes which have plate scales of 50 to 100 arcsec $\mathrm{mm}^{-1}$. With this equipment, variables of lower amplitude (say Bamplitude less than 0.75 magnitudes) are not found with any completeness. Surveys for RR Lyrae stars in globular clusters are usually made on reflector plates of better plate scale and variables of lower amplitude can be detected; in this case most of the c-type variables will probably be discovered. The technique of using the AF stars allows us to acheive a completeness for the discovery of the low amplitude variables in the field which should be at least as good as that in the well-searched globular clusters. 